\title{
Strength Improvement of Adhesively-Bonded Joints Using a Reverse-Bent Geometry
}

\author{
R. D. S. G. Campilho, A. M. G. Pinto, M. D. Banea, R. F. Silva \\ and L. F. M. da Silva
}

\begin{abstract}
Adhesive bonding of components has become more efficient in recent years due to the developments in adhesive technology, which has resulted in higher peel and shear strengths, and also in allowable ductility up to failure. As a result, fastening and riveting methods are being progressively replaced by adhesive bonding, allowing a big step towards stronger and lighter unions. However, single-lap bonded joints still generate substantial peel and shear stress concentrations at the overlap edges that can be harmful to the structure, especially when using brittle adhesives that do not allow plasticization in these regions. In this work, a numerical and experimental study is performed to evaluate the feasibility of bending the adherends at the ends of the overlap for the strength improvement of single-lap aluminium joints bonded with a brittle and a ductile adhesive. Different combinations of joint eccentricity were tested, including absence of eccentricity, allowing the optimization of the joint. A Finite Element stress and failure analysis in ABAQUS ${ }^{\circledR}$ was also carried out to provide a better understanding of the bent configuration. Results showed a major advantage of using the proposed modification for the brittle adhesive, but the joints with the ductile adhesive were not much affected by the bending technique.
\end{abstract}

\section{Keywords}

Bonded joint, structural adhesive, finite element analysis, strength prediction

\section{Introduction}

Adhesive bonded joints have become more efficient in the last few decades due to the developments in adhesive technology, which has resulted in higher peel 
and shear strengths, and also in allowable ductility up to failure. As a result of the reported improvement in the mechanical characteristics of adhesives, adhesive bonding has progressively replaced traditional joining methods such as fastening or riveting, allowing a big step towards stronger and lighter unions between components. Compared to these traditional techniques, adhesive bonded joints also benefit from smaller stress concentrations, absence of fretting between materials to be joined, improved fatigue behaviour, easier conformance to complex shapes, amongst many other factors. However, it is common knowledge that stress concentrations still subsist in bonded joints along the bond length owing to the gradual transfer of load between the two adherends in the overlap region (also known as differential straining along the overlap), especially in single-lap joints [1]. As a result, shear stresses concentrate at the overlap edges, with only a very small amount of load being carried in the central region. Peel stresses also develop in the same regions owing to the joint rotation and curvature of the adherends [2]. Both of these can be harmful to the structure, especially when using relatively brittle adhesives, which do not allow redistribution of stresses at the loci of higher concentrations, i.e., the overlap edges, leading to premature failures.

To overcome these limitations, considerable research has been carried out in recent years on the development of more efficient adhesively-bonded techniques that are able to suppress the concentrations of stresses as well as on adhesive technology [3-6]. One of the most commonly applied techniques is the use of adhesive fillets at the overlap edges. Fillets allow the redistribution of stresses in the mentioned regions and, as a result, they increase the strength of bonded unions [7-9]. Fillets usually extend over all the adherends thickness, minimizing peak peel and shear stresses at the overlap edges [10]. Rispler et al. [11] developed a numerical algorithm to find the optimal fillet shape in adhesively-bonded reinforced plates. In each iteration of the optimization process, the low stressed fillet elements were deleted in order to optimize their shapes. The optimal solution (a $45^{\circ}$ flat fillet) was achieved when all fillet representative elements were stressed by at least $20 \%$ of the structure maximum stress. A two-dimensional Finite Element study was published by Lang and Mallick [12], concerning the effect of the fillet shape on peel and shear stresses in a single-lap joint loaded in tension. Reductions in peel and peak shear stresses of 87 and $60 \%$ were achieved at the overlap edges using a curved fillet. These results are consistent with the work of Quaresimin and Ricotta [13], whose experimental data revealed that efficiency improvements from 11.6 to $25.2 \%$ could be achieved with a $45^{\circ}$ straight fillet, depending on the overlap length and surface condition of the carbon-epoxy adherends (with or without peel-ply).

Outer and inner tapering of the patches can also be effective in reducing peak peel stresses at the overlap edges [14-16], eventually increasing the load bearing capability of the repairs. Kaye and Heller [17] emphasized that patch outer tapering distributes the loads more uniformly between the laminates and patches, which reflects in a strength improvement of bonded structures. Hu and Soutis [18] showed that peak shear strains can be markedly reduced by increasing the adhesive thick- 
ness at the patch edges. Therefore, a joint with patches tapered from inside was considered to reduce stress concentrations in the adhesive layer and consequently to increase the joint strength. da Silva and Adams [19] studied for double-lap joints the effects of internal patch tapering and filleting on peel stresses and on the joint strength under varying temperatures. Stresses along the bondline greatly diminished with this modification under tensile loads. The experimental results showed that, as a rule, tapering and filleting increased the joint strength at ambient temperatures. At low temperatures, the differences were not significant.

Ganesh and Choo [20] evaluated Young's modulus grading of the adherends in single-lap joints under tension to reduce stress concentrations. Finite Element results showed a $20 \%$ reduction in peak shear stresses in the adhesive layer, concurrently with an increased load transfer in the central region of the bondline. Boss et al. [21] followed an alternate route, considering in addition to the aforementioned technique an edge chamfer to improve the joint strength. A reduction in peak shear stresses was achieved with modulus grading and chamfering. However, only the chamfering technique was able to reduce peel stresses. Ávila and Bueno [22] tested a wavy geometry (with a sinusoidal adherend shape at the overlap). This approach increased the joint strength by approximately $40 \%$, which was justified by the uniformity of shear and peel stress distributions in the adhesive layer. An identical solution was tested by Zeng and Sun [23], which showed that this technique allows a large improvement in load capacity of the joints, mainly due to the development of compressive through-thickness stresses at the edges of the overlap.

Campilho et al. [24] evaluated by Finite Elements coupled with cohesive modelling the tensile strength of adhesively-bonded single and double-strap repairs. Several geometric alterations, such as fillets, chamfering the patch outer and inner faces, plug filling and chamfering the outer and inner plate edges, were tested. For the single-strap repairs, the best results ( $26.8 \%$ strength improvement) were achieved by filleting the patch ends and chamfering the outer and inner edges of the adherends. Using the double-strap technique, the strength improvement was highest by using a flat fillet at the patch ends and plug filling with adhesive the gap between the adherends (strength improvement of 11.9\%).

The work of McLaren and MacInnes [25] is considered as the pioneering work on the subject of single-lap joints with a bent edge at the overlap for the optimization of stress distributions by elimination of the joints eccentricity. The bent modification to the lap joint with flat adherends was proposed and analysed by photoelasticity, showing the effectiveness of this technique to reduce stress gradients along the bondline. The most impressive results were attained for certain negative values of adherends eccentricity. Related studies performed a couple of decades later by Das Gupta and Sharma [26] and Das Gupta [27] led to similar conclusions, but considering the adherends bent outside the overlap region, i.e., keeping a constant thickness bondline. Sancaktar and Lawry [28] evaluated the use of single-lap joints with prebent adherends by photoelasticity, considering resin adherends bonded with a liquid plastic cement. Photoelasticity was used to experimentally ascertain the magnitude 
of tear stresses. Experimental testing also revealed that for the joint materials selected for the study, the failure strength of the joints could be increased up to $71 \%$, compared with the flat joint. Fessel et al. [29] performed an experimental and Finite Element study on tensile loaded steel single-lap joints, with emphasis on wavy and bent geometries. These modifications diminished peak peel and shear stresses at the overlap edges. The experimental tests showed strength improvements for the bent joint from 8 to $40 \%$, compared to a flat geometry.

In this work, a parametric study was performed on single-lap aluminium joints bonded with two adhesives, a brittle (Araldite ${ }^{\circledR}$ AV138) and a ductile one (Araldite ${ }^{\circledR}$ 2015), to evaluate the feasibility of bending the adherends at the ends of the overlap (configuration known as bent joint) for the strength improvement of these joints. The experimental study comprises different combinations of joint eccentricity, including absence of eccentricity, for strength optimization. A Finite Element stress and failure analysis in ABAQUS ${ }^{\circledR}$ was also carried out to provide a deeper insight into the effect of the bent configuration on the joint behaviour. Failure was predicted with two straightforward failure criteria, each one particularly suited to one of the adhesives, as they capture the essence of the respective failure process while giving acceptable results.

\section{Characterization of the Materials}

The aluminium alloy AW6082 T651 was selected for the adherends, characterized by a high tensile strength (340 $\mathrm{MPa}$ as specified by the manufacturer) obtained through artificial ageing at a temperature of approximately $180^{\circ} \mathrm{C}$ [30]. This specific alloy was chosen due to its wide use in Europe for several structural applications under different extruded shapes. The bulk stress-strain $(\sigma-\varepsilon)$ response of the aluminium adherends, obtained according to the ASTM-E8M-04 standard [31], is presented in Fig. 1. The aluminium alloy has a Young's modulus $(E)$ of $70.07 £ 0.83 \mathrm{GPa}$, a yield stress $\left(\sigma_{\mathrm{y}}\right)$ of $261.67_{ \pm} 7.65 \mathrm{MPa}$, a maximum strength $\left(\sigma_{\mathrm{f}}\right)$ of $324_{ \pm} 0.16 \mathrm{MPa}$ and a failure strain $\left(\bar{\varepsilon}_{\mathrm{f}}\right)$ of $21.7 q_{-} 4.24 \%$. The bilinear approximation of Fig. 1 was used for input in the simulations. The two

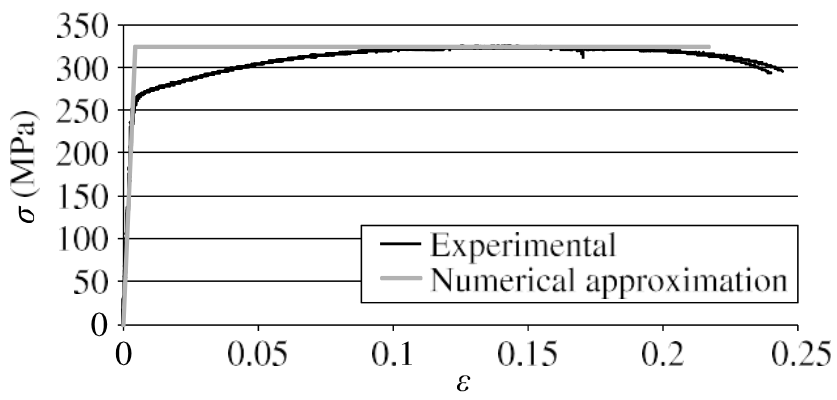

Figure 1. $\sigma-\varepsilon$ curves of the aluminium AW6082 T651 and respective approximation for the Finite Element analysis. 


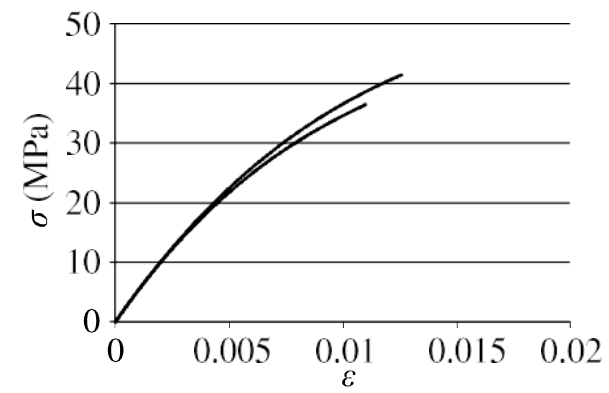

(a)

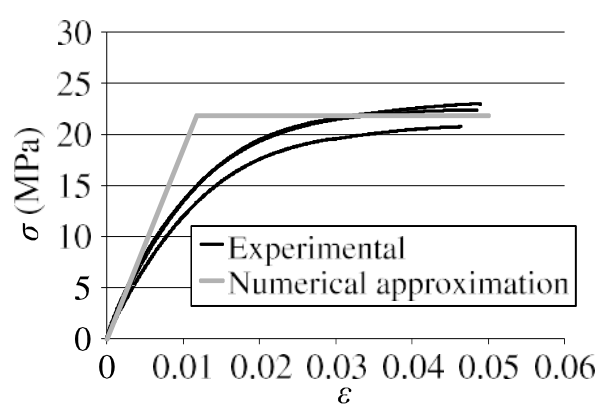

(b)

Figure 2. $\sigma-\varepsilon$ curves of the Araldite ${ }^{\circledR}$ AV138 (a) and Araldite ${ }^{\circledR} 2015$ with approximation for the Finite Element analysis (b).

adhesives, Araldite ${ }^{\circledR}$ AV138 and Araldite ${ }^{\circledR}$ 2015, were also characterized for subsequent input in the Finite Element analysis that will make possible the analysis of the results and comparison with the experiments. The tests were carried out under tension (mode I loading; bulk tests) and shear (mode II loading; Thick Adherend Shear Test (TAST)), which allowed the determination of the yield strengths and moduli in both loading modes. The bulk specimens for both adhesives were fabricated according to the French standard NF T 76-142 [32] to prevent the formation of porosities. Thus, the specimens were made of $2 \mathrm{~mm}$ plates, cured under pressure in a sealed mould, followed by machining to produce the dogbone shape described in the standard. The TAST tests followed the guidelines of the ISO 11003-2:1999 standard [33], using DIN Ck 45 steel for the adherends. Particular attention was paid to the surface preparation and bonding procedures to guarantee a cohesive failure of the adhesive, which followed entirely the specifications of the standard. Figure 2 shows, as an example, typical stress-strain curves in pure mode I of the Araldite ${ }^{\circledR}$ AV138 (a) and Araldite ${ }^{\circledR} 2015$ (b). For the Araldite ${ }^{\circledR} 2015$, a bilinear approximation was made for the subsequent Finite Element failure analysis. The difference between these two adhesives concerning the allowable ductility is notorious, as the AV138 is extremely fragile, while the 2015 undergoes large plasticization prior to failure. A higher deviation between specimens was also found for the AV138 since, due to its brittleness, it is more sensitive to fabrication defects [34]. The failure strength of the AV138 is nearly twice that of the 2015. Table 1 summarizes the data on these materials [34], which will be subsequently used for the Finite Element simulations and strength predictions. The initial yield strength was calculated for a plastic deformation of $0.2 \%$ for both adhesives.

\section{Experimental Work}

The eccentricity parameter of a single-lap joint, $K$, is defined as

$$
K=\frac{e}{t_{\mathrm{P}}+t_{\mathrm{A}}},
$$


Table 1.

Properties of the adhesives Araldite ${ }^{\circledR}$ AV138 and 2015

\begin{tabular}{lll}
\hline Property & AV138 & 2015 \\
\hline Young's modulus, $E(\mathrm{GPa})$ & $4.89 \pm 0.81$ & $1.85 \pm 0.21$ \\
Poisson's ratio, $v^{*}$ & 0.35 & 0.33 \\
Tensile yield strength, $\sigma_{\mathrm{y}}(\mathrm{MPa})$ & $36.49 \pm 2.47$ & $12.63 \pm 0.61$ \\
Tensile failure strength, $\sigma_{\mathrm{f}}(\mathrm{MPa})$ & $39.45 \pm 3.18$ & $21.63 \pm 1.61$ \\
Tensile failure strain, $\boldsymbol{\varepsilon}_{\mathrm{f}}(\%)$ & $1.21 \pm 0.10$ & $4.77 \pm 0.15$ \\
Shear modulus, $G(\mathrm{GPa})$ & $1.56 \pm 0.01$ & $0.56 \pm 0.21$ \\
Shear yield strength, $\tau_{\mathrm{y}}(\mathrm{MPa})$ & $25.1 \pm 0.33$ & $14.6 \pm 1.3$ \\
Shear failure strength, $\tau_{\mathrm{f}}(\mathrm{MPa})$ & $30.2 \pm 0.40$ & $17.9 \pm 1.8$ \\
Shear failure strain, $\gamma_{\mathrm{f}}(\%)$ & $7.8 \pm 0.7$ & $43.9 \pm 3.4$ \\
\hline
\end{tabular}

*Manufacturer's data.

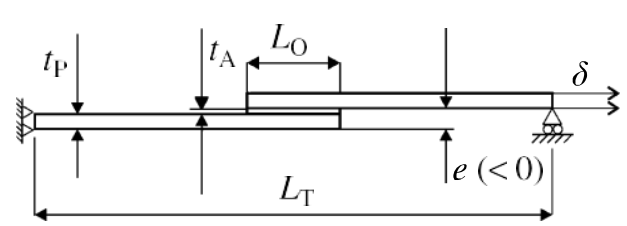

(a)

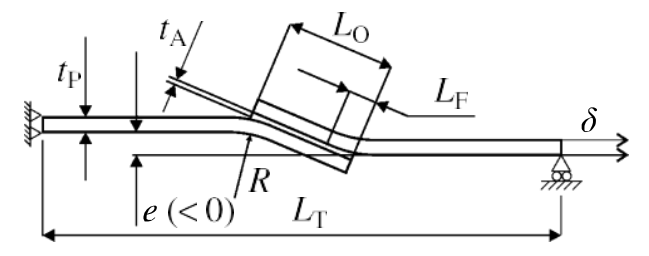

(b)

Figure 3. Geometry and dimensions of a single-lap joint with flat adherends, $K=1$ (a) and with reverse-bent geometry, $K=-1$ (b).

where $e$ is the absolute eccentricity between the adherends (measured offset between lower or upper adherend faces), $t_{\mathrm{P}}$ the adherend thickness and $t_{\mathrm{A}}$ the adhesive thickness. Figure 3 specifies the joint geometry and dimensions for a flat joint, $K=1$ (a) and bent joint with negative eccentricity, $K=-1$ (b). The chosen values for the dimensions were as follows: to $3 \mathrm{~mm}, t_{£} 0.2 \mathrm{~mm}$, variable bending radius $(R)$ to achieve a pre-defined size for the internal fillet length $\left(L_{ \pm \pm} 3 \mathrm{~mm}\right)$, overlap length $L_{\mathrm{O}}=20 \mathrm{~mm}$, total length between grips $L_{\mathrm{T}}=180 \mathrm{~mm}$, and varying values of $e$ to provide different values of $K$. Joints were tested between $K 1$ (single-lap joint with flat adherends) and $\underline{K} 1$, also considering a joint with perfectly aligned adherends $(K=0)$, based on evidence from previous works as the limiting range that can possibly yield a strength advantage over the flat geometry $[29,35]$. Following unanimous guidelines of published works on this matter $[29,36]$, the adherend curvature was kept within the overlap range (Fig. 3) to further provide a reduction of stress concentrations at the overlap edges, considering a variable value of $R$ that depends on $K$ to produce an internal fillet size of $15 \%$ of the overlap $\left(L_{\mathrm{F}} \underline{\underline{3}} \mathrm{~mm}\right)$. The chosen value of $t_{\mathrm{P}}$ was checked numerically before testing to prevent plastic straining in the adherends during loading that would render this modification ineffective by the elimination of the adherends curvature. The adherends were machined to the chosen dimensions and then manually bent 


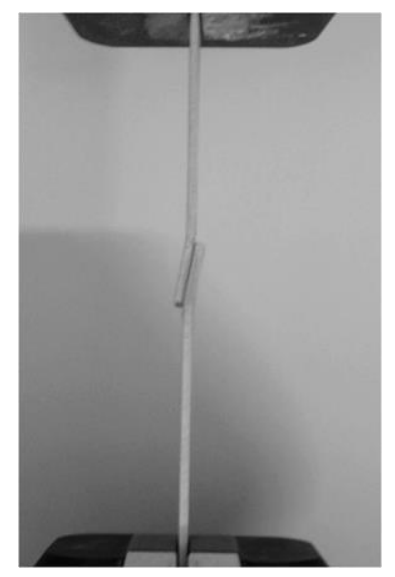

Figure 4. Specimen setup in the testing machine (Araldite ${ }^{\circledR} 2015 ; K=0$ ).

by plastic straining using an industrial press to the prescribed angle for each of the $K$ values. Bonding was carried out after grit blasting and acetone wiping using an apparatus for the correct alignment and placing of $0.2 \mathrm{~mm}$ diameter calibrated fishing lines at the overlap edges to guarantee the correct value of $t_{\mathrm{A}}$. A manual positioning method with a digital caliper was used for correct alignment. Tabs of different thicknesses were also bonded for a proper alignment in the testing machine. Curing of the specimens was achieved at room temperature according to the manufacturer's specifications for a complete curing. The tests were carried out in a Shimadzu AG-X 100 testing machine with a $100 \mathrm{kN}$ load cell, at room temperature and under displacement control $(0.5 \mathrm{~mm} / \mathrm{min})$. Four valid results were always provided for each condition. Figure 4 shows the specimen setup in the testing machine (Araldite ${ }^{\circledR} 2015 ; K \varrho$ ). For this particular configuration, no tabs were used since the adherends were perfectly aligned.

\section{Numerical Analysis}

A non-linear geometrical numerical analysis was performed in $\mathrm{ABAQUS}^{\circledR}$ using the aforementioned material properties (Table 1), neglecting the plastic deformation of the adherends induced by the bending procedure. Figure 5 depicts the meshes for the flat joint, $K=1$ (a) and reverse-bent joint with $\underline{K} 1$ (b). The joints were modelled without any symmetry conditions owing to the absence of vertical or horizontal symmetry. Restraining and loading conditions are visible in Fig. 3, consisting in clamping the joint at one of its edges and restraining vertically on the opposite edge, to faithfully reproduce the testing conditions [37,38], while this same edge is under a prescribed displacement $\left(\delta\right.$; Fig. 3 ). The joint was meshed by ABAQUS ${ }^{\circledR}$ $\mathrm{CAE}$ meshing algorithms from the user introduced seeding preferences (including bias effects), considering CPE8 elements for two-dimensional plane-strain conditions. The mesh was particularly refined at the overlap edges to accurately capture loci of stress concentrations [2]. The Finite Element analysis will allow comparison 


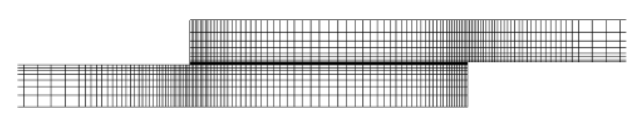

(a)

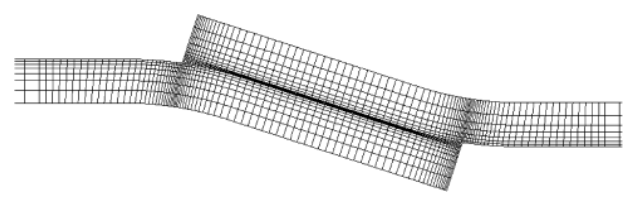

(b)

Figure 5. Finite Element mesh of a single-lap joint with flat adherends, $K=1$ (a) and with reverse-bent geometry, $K=-1$ (b).

of stress distributions along the adhesive mid-thickness between the different joint configurations tested and to explain the experimental results in terms of strength improvement. It will also be used in conjunction with two straightforward failure criteria, each one suited for one of the failure processes emerging from the characteristics of the adhesives, to predict the failure load of the joints. Due to the different behaviours of the two adhesives used, different criteria will be employed. The strength of the joints bonded with the brittle adhesive AV138 will be predicted using the Maximum Shear Stress Criterion [39]. This is an extremely simple method to apply, which states that the adhesive bondline fails when the maximum shear stress in the adhesive bond $\left(\tau_{\mathrm{y}}\right)$ attains the shear strength of the adhesive $\left(\tau_{\mathrm{f}}\right)$, i.e.,

$$
\tau_{\mathrm{y}}=\tau_{\mathrm{f}} .
$$

It is clear that this method suffers from the well-known mesh dependency issues if stresses are computed in singular or stress concentration regions [40], which is the present case, but it will be more than suitable to capture the influence of the value of $K$ on the joints strength, as the peak shear stresses at the overlap edges will be the leading factor for the joints failure [41]. On the contrary, this technique would definitely not be appropriate for the simulation of the joints bonded with the ductile adhesive 2015, since failure will occur largely after the beginning of yielding in the adhesive. Under these conditions, adhesives typically fail by global yielding [42, 43], considering that the entire bond has attained the yield stress of the adhesive, i.e.,

$$
P_{\mathrm{m}}=\tau_{\mathrm{f}} \times b \times L_{\mathrm{O}},
$$

where $P_{\mathrm{m}}$ is the failure load of the joint and $b$ the joint width. However, for the geometries tested in this work, the application of this criterion would lead to identical results irrespective of the value of $K$, as the bonding area is kept unchanged, with the variations in peel stresses emerging from the different values of $K$ not interfering with the results. To account for this difference, an elastic-perfectly plastic approximation to the experimental $\sigma-\varepsilon$ curves of Fig. 2(b) was considered for the simulations. The global behaviour of the adhesive, including the large shear component, was modelled using the von Mises yield criterion. $P_{\mathrm{m}}$ can be found when $\varepsilon$ anywhere in the adhesive attains the experimentally measured value of $\varepsilon_{\mathrm{f}}$, being considered that crack propagation leading to the joint failure occurs at this stage. Using this procedure, the reduction of peel stresses with adherends bending can be accounted for in thepredictions. 


\section{Results}

\subsection{Stress Analysis}

The following stress analysis was carried out considering the elastic properties of the adhesive AV138, and the results obtained will be on the basis of the analysis for both adhesives, although very small differences may occur due to the different values of $E$. Figure 6 compares normal longitudinal $\left(\sigma_{11}\right)$ stresses for the joints with $K=1$ (a), $K=0$ (b) and $K=-1$ (c), under an identical tensile displacement of $0.03 \mathrm{~mm}$. The load eccentricity owing to the offset of the adherends is a distinctive feature of traditional joints with flat adherends (Fig. 6(a)), causing a bending moment that reflects in the adherends transverse flexure [44] and respective $\sigma_{11}$ stress gradients in the thickness direction (ranging from tensile stresses near the bond to compressive stresses at the opposite face). Under these conditions, $\sigma_{11}$ stresses range from approximately 11 to $32 \mathrm{MPa}$. $\sigma_{11}$ stresses also increase in magnitude from the non-bonded region towards the bond, where tensile $\sigma_{11}$ stresses due to the axial and bending efforts sum [45]. Along the overlap, $\sigma_{11}$ stresses in each of the adherends diminish towards the respective adherend edge, due to the reduction of shear transfer length contributing to the axial loads within the adherend. For a bent joint with aligned adherends, $K=0$ (Fig. 6(b)), the resulting $\sigma_{11}$ stresses are significantly smaller than for the previous condition (varying from approximately 0 to $18 \mathrm{MPa}$ ), as the adherends bending is suppressed. As a result, $\sigma_{11}$ stresses are
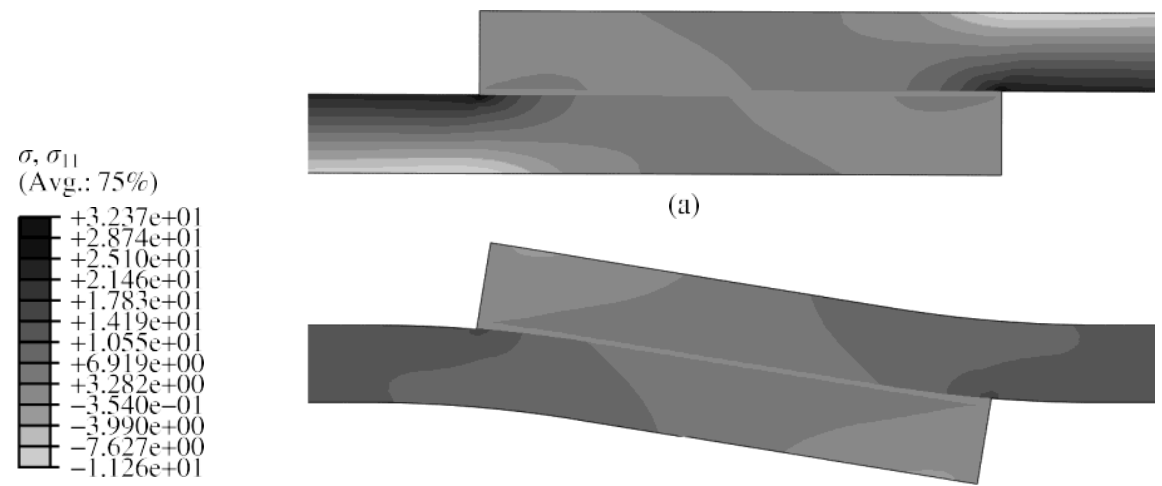

(a)

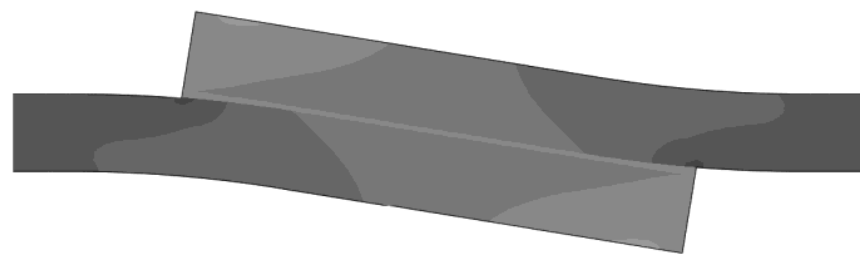

(b)

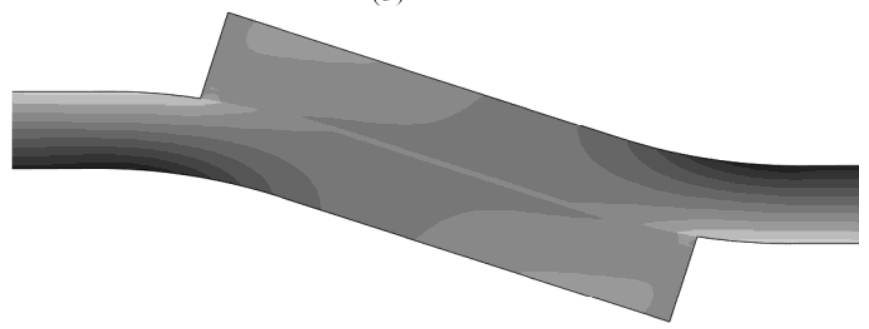

(c)

Figure 6. Longitudinal normal stresses (MPa) for the joints with $K=1$ (a), $K=0$ (b) and $K=-1$ (c). 
nearly constant in the adherends outside the bonding region. As far as $\sigma_{11}$ stresses are concerned, this is the most effective solution. Actually, for the reverse-bent geometry with $K \perp$ (Fig. 6(c)), an opposite bending to the flat geometry is found, giving rise to $\sigma_{11}$ stresses from approximately $10 \mathrm{MPa}$ (near the bond) to $25 \mathrm{MPa}$ (at the opposite face). As it was emphasized by Fessel et al. [29], the compressive stresses in the adherends near the overlap edges may help to reduce shear stresses at these regions, resulting in a more uniform distribution of shear stresses.

Figures 7 and 8 report on the through-thickness normal $(\sigma)$ and shear $(\tau)$ stresses, respectively, for the joints with the adhesive 2015 along the adhesive mid-thickness, as a function of the normalized overlap $\left(x / L_{0}\right)$. A normalization procedure was carried out, dividing $\sigma$ and $\tau$ stresses by $\tau_{\text {avg, }}$, the average shear stress along $L_{\mathrm{O}}$ for each value of $K$. For the flat joint $(K=1), \sigma$ peel stresses build up at the overlap edges in a very restricted region, owing to the square-edge geometry [46]. In the inner overlap region, these stresses are compressive, although smaller in magnitude than $\tau_{\text {avg. }}$. The classic shape of $\sigma$ peel stresses peaking at the overlap edges from compressive inner regions is mainly due to the already discussed asymmetry

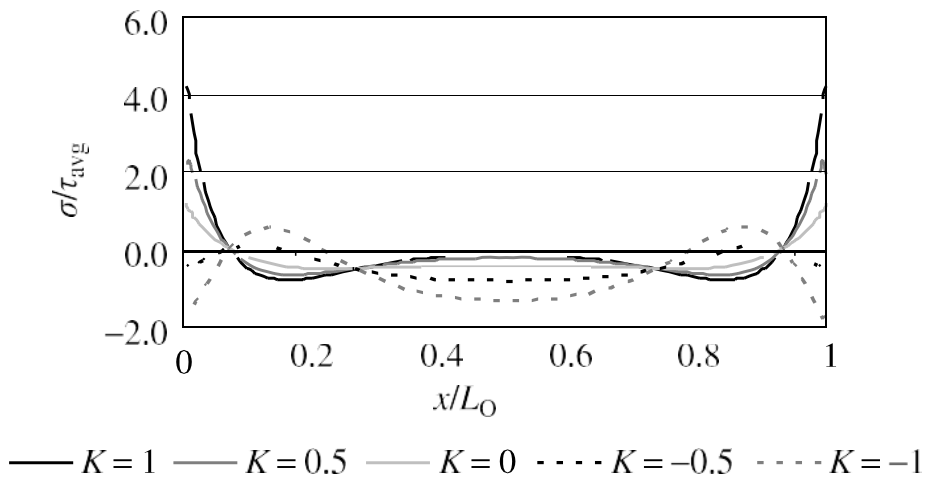

Figure 7. Through-thickness stress distributions at the adhesive layer mid-thickness for the different values of $K$.

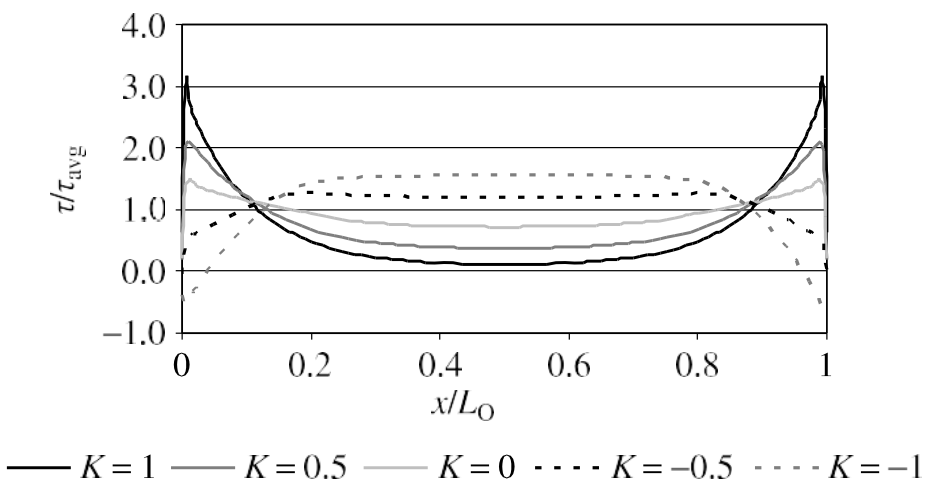

Figure 8. Shear stress distributions at the adhesive layer mid-thickness for the different values of $K$. 
of loading, yielding the transverse flexure of the joints [47]. This effect is more noticeable with lower stiffness adherends, which undergo larger flexure [2]. $\sigma$ peel singularities are usually regarded to significantly diminish the strength of adhesive layers, especially when using brittle adhesives, by inducing premature damage initiation at the overlap edges $[15,24]$. One of the main objectives of the pre-bent geometries is to prevent the adherend rotation and to suppress peak $\sigma$ peel stresses at the overlap edges, by relocating them towards the central region of the bondline. As it is evident from Fig. 7, this is gradually accomplished as $K$ varies between $K \pm$ to $K \quad 1$ anticipating a positive effect on the global behaviour of the joint. From $K \Theta, \sigma$ stresses become compressive at the overlap edges. Concurrently, the compressive $\sigma$ stresses near the overlap edges tend to vanish and a compressive region develops near the central region of the overlap. These results are consistent with the work of You et al. [35].

$\tau$ stresses for the flat joint are also consistent with the reported tendencies for single-lap joints, with a smaller load bearing potential in the overlap inner region and peaking towards the overlap edges [48]. This is caused by the differential deformation of each of the adherends along the overlap. In fact, the adherends are increasingly loaded from their free overlap edge towards the other overlap edge. As a result of this gradient, the practically unloaded free edges of each adherend slide relatively to the matching regions of the other adherend that endure high longitudinal deformations, causing $\tau$ peak stresses in those regions $[15,30]$. In the central region of the overlap these effects are cancelled, with $\tau$ stresses developing solely by the tensile pulling of the specimen. $\tau$ peak stresses are the leading cause for damage initiation at the overlap edges, affecting the strength of single-lap joints more severely for brittle adhesives, which do not allow plasticization at the overlap edges $[49,50]$. The increasing bending of the adherends at the overlap has an analogous effect to $\sigma$ stresses due to the reduction of the adherend differential straining [35]. Thus, $\tau$ peak stresses at the overlap edges become less significant with the increase of the adherends bending, up to $K=-0.5$, increasing again for $K=1$ owing to the appearance of compressive $\sigma_{11}$ stresses in the adherends near the overlap edges (Fig. 6) [29]. As a result of this modification, the shear loads get transferred by a larger region of the overlap, moving away from the overlap edges to the central overlap region [35]. This amendment, together with the change in $\sigma$ stresses, will probably prompt an increase in strength of the joints, especially for the adhesive AV138, whose brittleness will not support the redistribution of stresses in the bondline after the failure strength of the adhesive is attained at the overlap edges $[49,50]$.

\subsection{Test Results}

All fractures of the joints were due to cohesive failure of the adhesive, which testifies the effectiveness of the chosen adhesives and surface preparation method to bond the aluminium adherends. Equally, the joint behaviour was approximately linear up to failure (always occurring abruptly). Figures 9 and 10 show the load- 


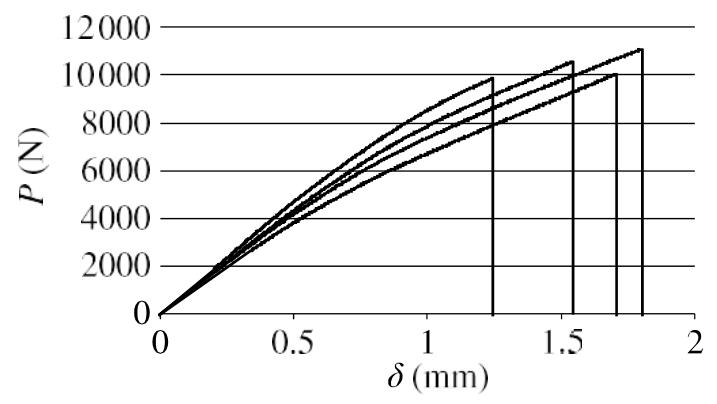

Figure 9. Experimental $P-\delta$ curves for the joints with $K=-0.5$ (Araldite ${ }^{\circledR}$ AV138).

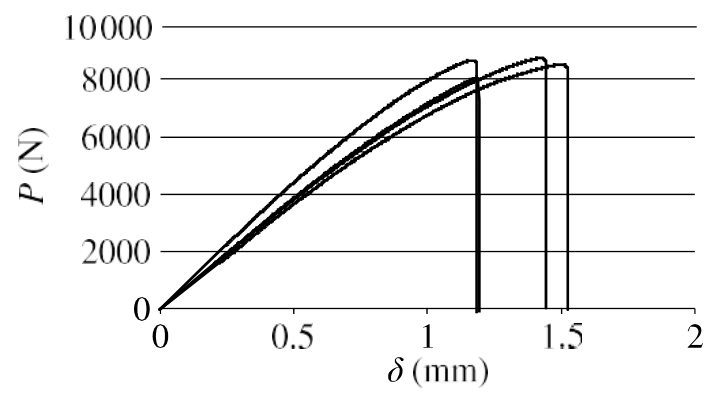

Figure 10. Experimental $P-\delta$ curves for the joints with $K=-1$ (Araldite ${ }^{\circledR} 2015$ ).

displacement $(P-\delta)$ curves for the joints with $K=-0.5$ for the adhesive AV138 and $K \pm$ for the adhesive 2015, respectively (corresponding to the best experimental results). In both cases, the $P-\delta$ relationship is approximately linear up to failure, although a progressive softening can be found beginning at specific values of applied $\delta$. The elimination or reduction of the transverse deflection effects existent in flat single-lap joints by using the bent geometry [35] is shown for the adhesive Araldite ${ }^{\circledR}$ AV138 in Fig. 11 (joint with flat adherends; $K$ 1), Fig. 12 (joints with aligned adherends, $K=0$ ) and Fig. 13 (reverse-bent joint; $K=-1$ ), by comparing the experimental deformation of the joints with the numerical simulations. The vertical reference lines testify the absence of transverse deflection for $K=0$.

\subsection{Strength Study}

The experimental $P_{\mathrm{m}}$ results for the different values of $K$ (Fig. 14) show that, notwithstanding the type of adhesive, the proposed technique can be recommended for the joint with flat adherends $(K=1)$. Figure 14 also permits evaluation of the deviation between tests by the vertical error bars linked to each value of $P_{\mathrm{m}}$ and comparison with the numerical predictions, based on the two criteria described in Section 4 used for each of the adhesives (the Maximum Shear Stress criterion for the adhesive AV138 and the von Mises/ $\varepsilon_{\mathrm{f}}$ criterion for the adhesive 2015). Results are acceptable in view of the simplified criteria employed. The maximum percentile 

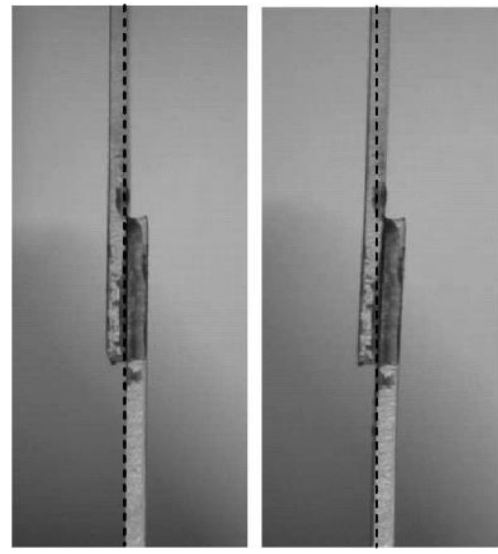

(a)

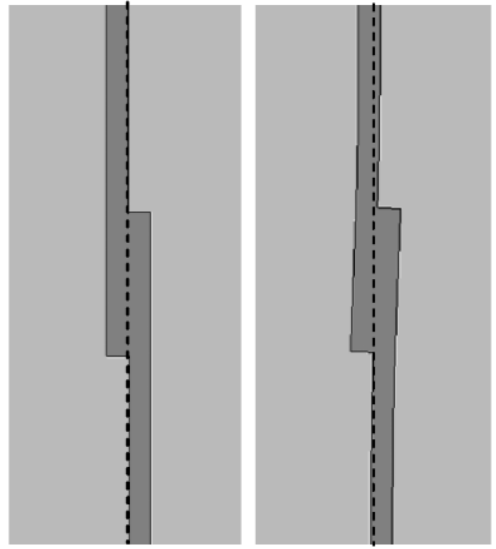

(b)

Figure 11. Experimental (a) and numerical (b) representation of a single-lap joint $(K=1)$ bonded with the adhesive Araldite ${ }^{\circledR}$ AV138 at the beginning of the test (left) and shortly before failure (right).

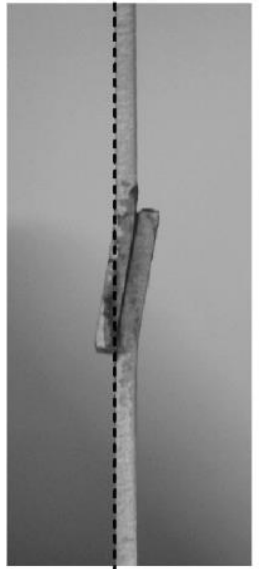

(a)

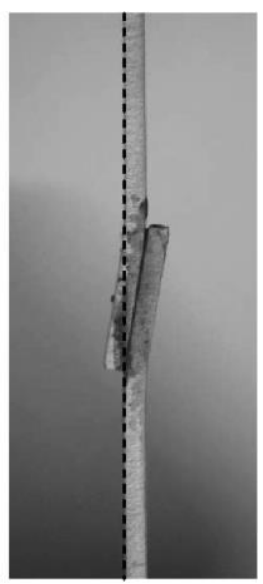

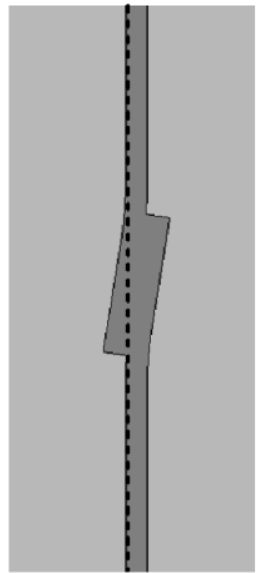

(b)

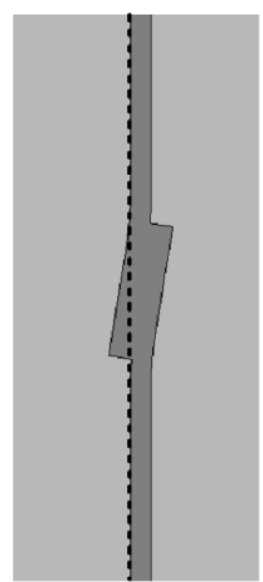

Figure 12. Experimental (a) and numerical (b) representation of a single-lap joint $(K=0)$ bonded with the adhesive Araldite ${ }^{\circledR}$ AV138 at the beginning of the test (left) and shortly before failure (right).

strength improvement is of approximately $162 \%$ for the brittle adhesive AV138 and $27 \%$ for the ductile adhesive 2015 . The reason for this difference will be discussed in the following. In terms of strength dependence with $K$, this is much more evident for the brittle adhesive AV138, for which a notorious improvement is gradually found from $K=1$ up to $K=0.5$ (approximately $162 \%$ ), which then slightly decreases with further bending the adherends $(K 1)$. On the contrary, with the ductile adhesive 2015, the reverse-bent geometry with $K=-1$ was shown to be the most effective, although results are identical between $K \quad 0$ and $K_{-}$. In contrast to the brittle adhesive, only a modest strength improvement was found (approximately $27 \%$ for the joints with $K=-1$ ). 


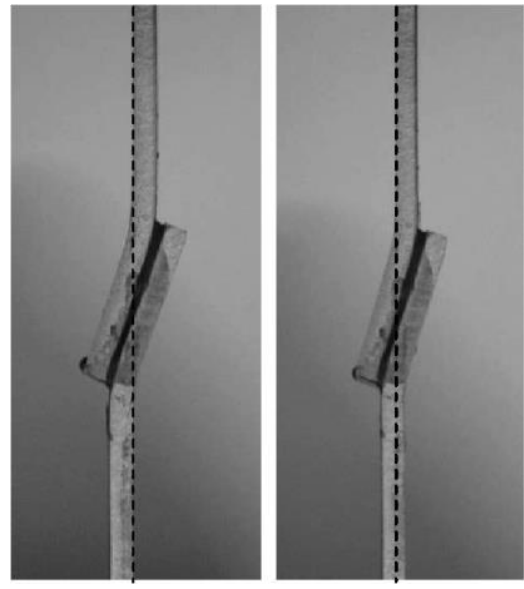

(a)

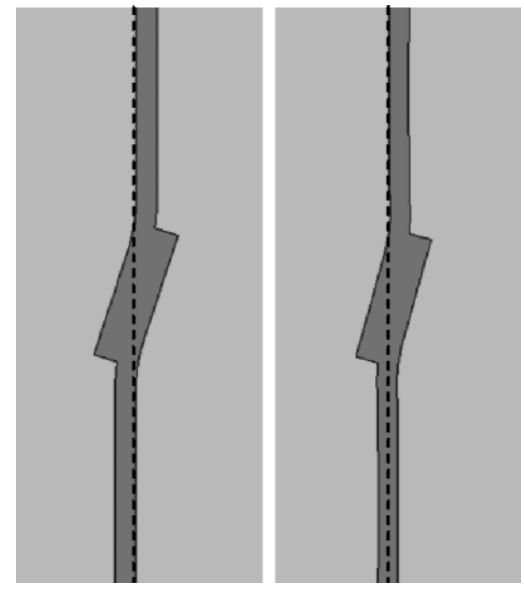

(b)

Figure 13. Experimental (a) and numerical (b) representation of a single-lap joint $(K=-1)$ bonded with the adhesive Araldite ${ }^{\circledR}$ AV138 at the beginning of the test (left) and shortly before failure (right).

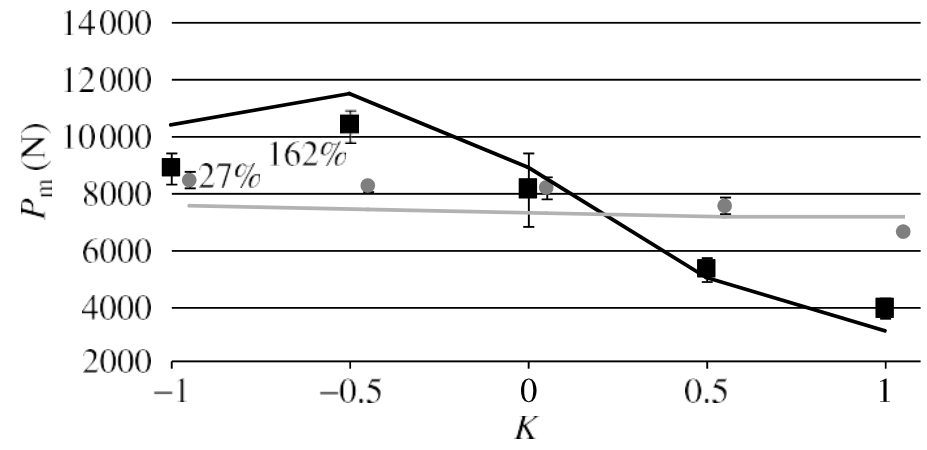

- AV138 experimental 2015 experimental — AV138 numercal — 2015 numerical

Figure 14. $P_{\mathrm{m}}$ as a function of $K$ for the joints bonded with Araldite ${ }^{\circledR}$ AV138 and 2015.

The non-consistency of strength improvement between the brittle and ductile adhesives is supported by the obvious difference in ductility. In fact, brittle adhesives such as the AV138 are extremely sensitive to peak stresses at the overlap edges since they do not allow plasticization in these regions and failure takes place once the strength of the adhesive is attained $[49,50]$. As a result, the large improvement of $P_{\mathrm{m}}$ with the reduction of $K$ is linked to the concurrent reduction of $\sigma$ and $\tau$ peak stresses (Figs 7 and 8). A reduction in $P_{\mathrm{m}}$ is then found for $K=-1$, which is related to the slight increase of $\sigma$ peel and $\tau$ stresses that, although not being significant for $K=-1$, weaken the joint compared to the joint with $K=-0.5$ [35]. This result is consistent with the early study by Greenwood et al. [51], which showed experimentally that, for similar joints but with thicker substrates, a value of $K$ of nearly -0.5 was recommended to achieve the highest joint strength. On 
the other hand, extremely ductile adhesives such as the 2015 in bonded joints fail under global yield conditions [52]. This means that when the peak stresses are attained at the overlap edges these regions undergo plasticization while the inner region of the bond, lightly loaded at this stage, starts to become loaded [41, 43]. As a consequence, at the time of failure the inner region of the bond is already under considerable load, which in turn renders the proposed modification not so useful. Actually, a maximum strength improvement of only approximately $27 \%$ between $K=0$ and $K=1$ was found. The results of Fig. 14, however, should not be viewed as absolute, since many different variables affect the strength improvement of the joints, one of which being the yielding of the adherends. Actually, for the adherends material used in this work, no further yielding than that induced in the fabrication process was detected for any of the joint configurations. However, adherends with smaller yield points could lead to premature failures at the overlap edges for joints with brittle adhesives, as the adhesive would not withstand the large deformations at the overlap edges [29]. On the other hand, it should be noted that reverse-bent joints suppress peak stresses in the adhesive and reduce yielding of the adherends, which in turn enables the use of more brittle adhesives even for adherends with smaller yield strengths. The steady improvement for the joints bonded with the adhesive 2015 in $P_{\mathrm{m}}$, from $K=1$ to $K=0$, can be attributed to the corresponding reduction in peel stresses (Fig. 7). In the work of Fessel et al. [29], the bent modification showed improvements in joint strength between 8 and $40 \%$ compared to the flat lap-shear joint, depending on the adherends and adhesive. However, the maximum strength was achieved with perfectly aligned adherends $(K=0)$.

Generally, the optimum value of $K$ depends on the material properties, ductility of the adhesive and, most importantly, ductility of the adherends. Previous studies $[29,36]$ addressed this subject, showing that adherends with low yield strengths require smaller values of $K$ to prevent plasticity of the adhesive near the overlap edges, thus avoiding a premature failure. Actually, with low strength adherends, the joints undergo large rotation and bending, and as a result failure will probably occur near the overlap edges by excessive metal straining, whilst only the inner region of the bond fails cohesively. By using the reverse-bent joint, the region of cohesive failure can be extended due to the reduction of adherend yielding. Using adherends with higher strengths, the values of $P_{\mathrm{m}}$ are also higher due to the reduction of adherends yielding.

\section{Concluding Remarks}

A study was carried out on single-lap aluminium joints bonded with two adhesives, a brittle (Araldite ${ }^{\circledR}$ AV138) and a ductile one (Araldite ${ }^{\circledR}$ 2015), to evaluate the feasibility of bending the adherends at the ends of the overlap (configuration known as bent joint) to improve the strength of single-lap joints. Different combinations of joint eccentricity were tested, including absence of eccentricity, allowing optimization of the joint. A Finite Element stress analysis in ABAQUS ${ }^{\circledR}$ was also carried 
out to provide a better understanding of the bent geometry, in conjunction with two straightforward failure criteria, one for each adhesive, which capture the essence of the respective failure process while giving acceptable results. The stress analysis, by providing through-thickness normal and shear stress distributions along the bondline, and also longitudinal normal stresses in the adherends, provided some insight into the effect of the proposed technique on the joint behaviour. As for longitudinal axial stresses, the joints with $K=0$, i.e., perfectly aligned adherends, were found to be most effective as the concentrations near the overlap edges were suppressed, which prevents local adherend yielding and reduces differential straining. Bending of the adherends also showed a positive effect on peak peel stresses at the overlap edges that gradually diminished by reducing the value of $K$ to 1 . The bending technique also reduces the adherend differential straining, and peak shear stresses become less significant at the overlap edges with the increase of the adherends bending up to $K=-0.5$, slightly increasing for $K=-1$. As a result of the improved stress distributions, the strength of the joint increased, especially for the adhesive AV138, whose brittleness leads to a fragile fracture as soon as the adhesive bond attains the failure strength at its edges. The strength study showed that, notwithstanding the type of adhesive, decreasing $K$ to 1 should be recommended for the condition of flat adherends $\left(K \_1\right)$. The maximum percentile strength improvement for the joint with flat adherends was approximately $162 \%$ for the brittle adhesive Araldite ${ }^{\circledR}$ AV138 and 27\% for the ductile adhesive Araldite ${ }^{\circledR} 2015$. The strength of the joints was found to be much more dependent on $K$ for the brittle adhesive AV138, which can be explained by its brittleness. In fact, brittle adhesives such as the AV138 are extremely sensitive to peak stresses as they do not allow plasticization. As a result, the major improvement in the failure load with the reduction of $K$ is closely related to the reduction in peak peel and shear stresses in the bondline. On the other hand, extremely ductile adhesives such as the 2015 in bonded joints fail under global yielding conditions. As a result, the improvement in strength is not so significant. The numerical predictions of failure load, taking advantage of the two straightforward criteria that capture the essence of the failure process, were within the range of the experiments.

\section{Acknowledgement}

The authors would like to thank the Portuguese Foundation for Science and Technology for supporting the work presented here.

\section{References}

1. R. D. S. G. Campilho, M. F. S. F. de Moura and J. J. M. S. Domingues, Int. J. Solids Struct. 45, 1497 (2008).

2. A. M. G. Pinto, A. G. Magalhães, R. D. S. G. Campilho, M. F. S. F. de Moura and A. P. M. Baptista, J. Adhesion 85, 351 (2009).

3. R. D. Adams and N. A. Peppiatt, J. Strain Anal. 9, 185(1974). 
4. A. A. Baker, Composite Struct. 2, 153 (1984).

5. A. A. Baker and R. Jones, Bonded Patch Repair of Aircraft Structures. Martinus Nijhoff, Dordrecht (1988).

6. H. Zhang, J. Motipalli, Y.C. Lam and A. Baker, Composites Part A 29, 1464(1998).

7. M. Y. Tsai and J. Morton, Composite Struct. 32, 123(1995).

8. I. A. Ashcroft, M. M. Abdel Wahab, A. D. Crocombe, D. J. Hughes and S. J. Shaw, Composites Part A 32, 45 (2001).

9. F. J. P. Chaves, L. F. M. da Silva and P. M. S. T. de Castro, J. Mater.: Design Appl. 222, 159 (2008).

10. P. T. Cheuk and L. Tong, Composites Sci. Technol. 62, 1079 (2002).

11. A. R. Rispler, L. Tong, G. P. Steven and M. R. Wisnom, Int. J. Adhesion Adhesives 20, 221 (2000).

12. T. P. Lang and P. K. Mallick, Int. J. Adhesion Adhesives 18, 167(1998).

13. M. Quaresimin and M. Ricotta, Composites Sci. Technol. 66, 176 (2006).

14. F. Mortensen and O. T. Thomsen, Composites Sci. Technol. 62, 1011 (2002).

15. R. D. S. G. Campilho, M. F. S. F. de Moura and J. J. M. S. Domingues, Composites Sci. Technol. 65, 1948 (2005).

16. G. Kress, P. Naeff, M. Niedermeier and P. Ermanni, Composite Struct. 73, 196(2006).

17. R. H. Kaye and M. Heller, Int. J. Adhesion Adhesives 22, 7 (2002).

18. F. Z. Hu and C. Soutis, Composites Sci. Technol. 60, 1103 (2000).

19. L. F. M. da Silva and R. D. Adams, Int. J. Adhesion Adhesives 27, 227 (2007).

20. V. K. Ganesh and T. S. Choo, J. Composite Mater. 36, 1757 (2002).

21. J. N. Boss, V.K. Ganesh and C. T. Lim, Composite Struct. 62, 113 (2003).

22. A. F. Ávila and P. O. Bueno, Int. J. Adhesion Adhesives 24, 407 (2004).

23. Q. Zeng and C. T. Sun, AIAA J. 39, 1991 (2001).

24. R. D. S. G. Campilho, M. F. S. F. de Moura and J. J. M. S. Domingues, Int. J. Adhesion Adhesives 29, 195 (2009).

25. A. S. McLaren and I. MacInnes, Brit. J. Appl. Phys. 9, 72 (1958).

26. S. Das Gupta and S. P. Sharma, ASME Publication 75, 18(1975).

27. S. Das Gupta, ASME Publication 79, 4(1979).

28. E. Sancaktar and P. O. Lawry, J. Adhesion 11, 233 (1980).

29. G. Fessel, J. G. Broughton, N. A. Fellows, J. F. Durodola and A. R. Hutchinson, Int. J. Adhesion Adhesives 27, 574 (2007).

30. P. M. G. P. Moreira, A. M. P. de Jesus, A. S. Ribeiro and P. M. S. T. de Castro, Theor. Appl. Fract. Mech. 50, 81 (2008).

31. ASTM-E8M-04. Standard test methods for tension testing of metallic materials (Metric) (2004).

32. NF T 76-142. Méthode de preparation de plaques d'adhésifs structuraux pour la réalisation d'éprouvettes d'essai de caractérisation (1988).

33. ISO 11003-2:1993(E). Adhesives-determination of shear behavior of structural bonds, part II: Thick Adherend Tensile Test method(1993).

34. L. F. M. da Silva, R. A. M. da Silva, J. A. G. Chousal and A. M. G. Pinto, J. Adhesion Sci. Technol. 22, 15 (2008).

35. M. You, Z. Li, X. L. Zheng, S. Yu, G. Y.Li and D. X. Sun, Int. J. Adhesion Adhesives 29, 280 (2009).

36. M. You, S. Yu, H. Z. Yu, Z. Li and W. J. Liu, Key Eng. Mater. 348, 273 (2007).

37. V. K. Goyal, E. R. Johnson and V. K. Goyal, Composite Struct. 82, 434 (2008).

38. J. J. Radice and J. R. Vinson, Composites Sci. Technol. 68, 376 (2008).

39. X. Liu and G. Wang, Composite Struct. 81, 3312007.

40. S. Feih and H. R. Shercliff, Int. J. Adhesion Adhesives 25, 47 (2005). 
41. M. D. Banea and L. F. M. da Silva, J. Mater.: Design Appl. 224, 51 (2010).

42. R. D. Adams, J. Comyn and W. C. Wake, Structural Adhesive Joints in Engineering, 2nd edn. Chapman \& Hall, London (1997).

43. M. D. Banea and L. F. M. da Silva, J. Adhesion 85, 261 (2009).

44. R. B. Heslehurst, Int. J. Adhesion Adhesives 19, 133 (1999).

45. R. D. S. G. Campilho, Modelação da Execução de Reparações em Materiais Compósitos, MSc Thesis, Department of Mechanical Engineering and Industrial Management, Engineering Faculty of Porto University, Porto, Portugal (2005).

46. J. Radice and J. Vinson, Composites Sci. Technol. 66, 2528(2006).

47. J. M. Ferreira, H. Silva, J. D. Costa and M. Richardson, Composites Part B 36, 1 (2005).

48. Q. Luo and L. Tong, Int. J. Solids Struct. 44, 2349(2007).

49. L. F. M. da Silva and M. J. C. Q. Lopes, Int. J. Adhesion Adhesives 29, 509 (2009).

50. L. D. R. Grant, R. D. Adams and L. F. M. da Silva, Int. J. Adhesion Adhesives 29, 405 (2009).

51. L. Greenwood, T. R. Boag and A. S. McLaren, in: Proceedings of International Conference, Adhesion: Fundamentals and Practice, University of Nottingham (1966).

52. A. D. Crocombe, Int. J. Adhesion Adhesives 9, 145 (1989). 